\title{
S-A-2. Evaluation of the Stochastic Study for Acute Head Injury
}

\author{
Kyozo Ando, Masayuki Matsushima, Toyoshiro Yamamoto, Hiroshi Tsuji, \\ Yoichi SuzukI, Shiro WaGa and Hiroshi OKa \\ Neurosurgical Department of Osaka Red Cross Hospital
}

Applying 203 acute head injury cases treated at the Osaka Red Cross Hospital for the past four years, "stochastic study for acute head injury" reported by Sano et al. of the Tokyo University was evaluated.

Our cases included 43 epidural hematoma cases, 46 subdural hematoma cases, 17 intracerebral hematoma cases, 54 combined intracranial hematoma cases and 43 non-hematoma cases. All of them satisfied following three conditinns: 1) closed head injury cases at the age over fifteen years, 2) acute head injury cases examined within three days, 3 ) head injury cases with moderate or severe consciousness disturbance.

Hematoma index was justified in 73.9 per cent of all cases, if we considered of the kind of hematoma the hitting rate remained as low as 50 per cent.

Hematoma side index proved in 87.6 per cent in our cases excluding bilateral hematoma cases ( 32 cases) or the cases, in which intracranial hematoma was denied stochastically.

Hematoma prognosis index was verified in 61.3 per cent of cases.

Our results may suggest that clinical features of acute head injury cases of the emergency hospital such as the Osaka Red Cross Hospital are quite different from those of the Tokyo University.

In addition to the stochastic study, we also reported acute head injury cases, in which extravasation of the contrast media or so-called non-filling phenomenon was observed angiographically.

\section{S-A-3. Re-evaluation of Araki's Classification of Head Injury}

- Chiefly on the IIIrd and IVth Types-

\author{
Kazuki SaKata, Takashi Yamamura, Yoshitomo Kashiki \\ and Takao Taketomo \\ 2nd Surgical Division, Gifu University School of Medicine
}

Dr. Araki's classification, now widely used in this country, is not only useful for evaluation of severity of head injuries, but also for obtaining the indication 
for operative treatment in the IVth type (the inracranial hemorrhage type). In the present situation of head injury treatment, cases in which the IVth type diagnosis is followed immediately, or simply after echoencephalography, by exploratory trephination or craniotomy are limited to a certain ultra-emergency cases. Generally carotid angiography precedes the operation. Carotid angiography, however, is not an innocuous examination in head injury cases. Our experiment in a brain edema dog showed that intracarotid injection of radiopaque medium (Conray) caused, after a transient increase, a decrease of cerebral blood flow and a rise of cerebrospinal fluid pressure, thus indicating unfavorable effect of carotid angiography. For the purpose of finding out on which occasion emergency carotid angiography is indicated, incidence of intracranial hematoma anl operative prognosis were examined in 137 acute and subacute head injury cases, mainly consisting of Araki's IIIrd (the cerebral contusion type) and IVth types. The cases were classified according to clinical courses of consciousness disturbance (C), subjective symptoms (S) and focal symptoms (F) into "O" group having asymptomatic interval, "1" group having relatively asymptomatic interval, "2" group showing continuous severe symptoms until operation or death and " 3 " group showing gradual alleviation. These cases were then made into 4 groups: (1) Araki's I, II-type with IV-type nuance, (2) IV-type with III-type nuance, (3) typical IV-type and (4) typical III-type. It was found that the incidence of intracranial hematoma was $64 \%$ in (3), $50 \%$ in (2) and $19 \%$ in (4). There were 2 hematoma cases in all 3 cases of (1). The incidence of hematoma in $\mathrm{C}_{2}$ group, the most severe cases in (4), was $67 \%$, but the operative prognosis was generally poor. The incidence of hematoma in $\mathrm{C}_{1}$ group, which belonged to (2) and showed a relatively lucid interval, was fairly high, but the operative prognosis was generally poor like the previous one. The operative prognosis in (3) was fairly good. In conclusion, Araki's classification will continue to be useful for diagnosis of head injury cases, but the following supplements will be considered. 1) All of the IVth type cases have an indication for emergency angiography; 2) Cases of the Ist type (the simple type) and the IInd type (the cerebral conclusion type), showing acutely aggravated subjective symptoms, have a relative indication for emergency angiography; 3 ) Severe cases of the IIIrd type have a relative indication for emergency angiography, but it should be known that there is a possibility of exacerbation following angiography and operative prognosis is relatively poor. 